\title{
Forensic Analysis of Offline Signatures Using Multilayer Perceptron and Random Forest
}

\author{
Abdul Salam Shah ${ }^{i *}$, Masood Shah ${ }^{2}$, Muhammad Fayaz ${ }^{3}$, Fazli Wahid ${ }^{4}$, Hira \\ Khalid Khan ${ }^{5}$ and Asadullah Shah ${ }^{6}$ \\ ${ }^{1,2,5}$ SZABIST, Islamabad, Pakistan \\ ${ }^{3}$ University of Malakand, KPK, Pakistan \\ ${ }^{4}$ Universiti Tun Hussein Onn, Malaysia \\ ${ }^{6}$ International Islamic University Malaysia (IIUM), Malaysia \\ I*shahsalamss@gmail.com, 22engg.cisco@gmail.com, ${ }^{3}$ hamaz_khan@yahoo.com, \\ ${ }^{4}$ wahid_uomian@hotmail.com, ${ }^{5}$ somefragrance6@gmail.com, \\ ${ }^{6}$ asadullah@iium.edu.my
}

\begin{abstract}
Forensic applications have great importance in the digital era, for the investigation of different types of crimes. The forensic analysis includes Deoxyribonucleic Acid (DNA) test, crime scene video and images, forged documents analysis, computer-based data recovery, fingerprint identification, handwritten signature verification and facial recognition. The signatures are divided into two types i.e. genuine and forgery. The forgery signature can lead to the huge amount of financial losses and create other legal issues as well. The process of forensic investigation for the verification of genuine signature and detection of forgery signatures in law related departments has been manual and the same can be automated using digital image processing techniques, and automated forensic signature verification applications. The signatures represent any person's authority so the forged signatures may also be used in a crime. Research has been done to automate the forensic investigation process, but due to the internal variations of signatures, the automation of signature verification still remained a challenging problem for researchers. In this paper, we have further extended previous research carried out in [1-2] and proposed a Forensic signature verification model based on two classifiers i.e. Multilayer Perceptron (MLP) and Random Forest for the classification of genuine and forgery signatures.
\end{abstract}

Keywords: Crime Investigation, Forensic Investigation, Forensic Signature, Forgery Signature, Image Processing, Random Tree, Signature Verification

\section{Introduction}

The signatures represent the authority of a person, and same can be used for illicit purposes. In the criminal sense, forged signature can be used to change the decision of any authority and impose an illegal decision. In the digital era, the automatic signature verification system will be very valuable in the field of Forensics. The signatures are mainly classified into two major groups i.e. On-line and Off-line. The authentication of the signature of a person is divided into two classes, i.e. genuine and forgery, the forgery signatures are further divided into Random Forgery, Simple Forgery and Skilled Forgery [1]. The importance of the signatures as identity has increased the chances of forgery, and unfortunately, there is no established method to verify the authenticity of signatures in an automated manner [2].

The development of new Forensic techniques based on digital image processing have much influence on Forensic Science. These techniques can be divided into automatic and 
interactive algorithms depending on the input, if user input or surveillance is necessary or not [3]. Forensic science techniques are used for investigation and establishment of facts and figures, and in relation to crime and law Forensic, it is an art and science, in which different type of analysis is involved. The basic purpose of Forensic science is to analyze everything that can be presented in court as evidence. If we talk about the scope of Forensic Science, the video on the crime scene, images of the crime scene, fingerprint identification, computer-based data recovery, facial identification, Deoxyribonucleic Acid (DNA) test, and signature matching, all are covered under the scope of Forensic Science [4-6].

The process of finding similarities, or the person's identity in the forensic signature is more difficult due to the probability of difference between the same person's signatures. The signer's psychophysical state is also reflected in signatures, so a complex theory is needed to identify the ink repository, psychophysical state, and the purpose of the signature [7]. The relation between Image Processing and Forensic Science is not only to check documents forgery. The image processing can also be used in other crime investigation application, to examine the crime scene photograph by image enhancement and image restoration and matching of facial identification of criminal [8].

The computer scientist, researchers, and handwriting experts are working in the area of Forensic signature verification. The signature image consists of thousands of pixels and for processing, we manipulate these pixels after applying mathematical operations. The steps of image processing techniques are mostly the training of algorithm or classifier to save the key points of an image in a mathematical way [8]. The comparison of the mathematical points with the new image under consideration. If the results are same or meet the threshold, then both images are genuine signed and if not then declared as forged. It is very difficult to find out the accurate threshold for detecting accurate value.

In this paper, we have used Multilayer Perceptron (MLP), and Random Forest as classifiers for the offline signature verification. Researchers have used MLP, for the signature verification, but very few have used Random Forest.

The rest of the paper is organized as, the Section 2, contain literature review, Section 3 proposed model, Section 4 contain the implementation of the model. Section 5 contains experimentation and results, Section 6 contain discussion and finally, the conclusion of the study is provided in Section 7.

\section{Literature Review}

Jena et al., in [3], used feature Points Extraction Method for the verification of signatures and Euclidian distance for classification. The horizontal splitting was carried out with a separate algorithm. The proposed technique has some disadvantages as another similar technique using 12 features, provided 14.58 False Rejection Rate (FRR), and 16.63 False Acceptance Rate (FAR), but the proposed technique with 60 points provided 20.83 FRR and 2.08 FAR which is not promising as compared to the features used by both techniques. Although the proposed technique is more reliable and efficient and provides accurate results, but it requires heavy calculation as compared to other techniques.

Bansal et al., in [7], proposed a contour matching algorithm for the tracking of signature patterns. The critical points and its physical significant with Polyfit Function was used for the signature verification. The signature images were converted to binary images using modified Niblack algorithm. A $5 * 5$ morphological filter was applied to remove the noise, and for the orientation of the images, the Principal Component Analysis was used. Euclidean distance has been used for the determination of position and orientation of the signature. The proposed technique has the major drawback that it has no universal applicability as the results depend upon the preprocessing and the wrong preprocessing may lead to unexpected results. 
Liu et al., in [9], used Dynamic Time Wrapping (DTW) algorithm to find out the best alignment of variable length sequences. The DTW was used for calculating the intra variance distance for the classification of the signature into genuine and forgery. The smoothening of the signature images has been carried out with the Gaussian filter. The use of variance for signature matching is better for accurate results. The deviation between real signatures is very less as compared to the deviation of real-forged signatures. The DTW is a more reliable and efficient technique that provides accurate results.

Serdouk et al. in [10], proposed Gradient Local Binary Pattern (GLBP) features for the characterization of offline signatures, with Support Vector Machine (SVM) classifier. Local Binary Pattern (LBP) neighborhoods are used for the computation of histogram of the signature image. They have considered eight neighborhoods for the image histogram calculation.

Bertolini et al. in [11], identified new feature set containing curvature of the most important segments of signature images. The Bezier curve was simulated and the features of signature images were extracted from that curve.

Miguel-Hurtado et al., in [12], continued their previous work of combining Dynamic Time Warping (DTW) and Gaussians Mixture Modeling (GMM) algorithms. The Dynamic Time Warping algorithm is used for nonlinear time $\mathrm{X}$-axis alignment and to impact minimization from other handwriting. Gaussian Mixture Modeling is used for the parametric statistical model and to identify the pattern of application. The mixture of DTW and GMM is the result of information position and pressure the DTW algorithm is used to obtain X-coordinate and $\mathrm{Y}$ coordinates in which wrapping path calculated to align the signals and the total distance between subsequence. For the verification, an MCYT database was used. The proposed algorithms have the EER of $2.40 \%$.

\section{Proposed Model}

The basic purpose of this research is to find out the optimal solution for automated identification/verification of forged and the original signature, using digital image processing techniques. The general process for a signature verification system is as under:

Signature Database

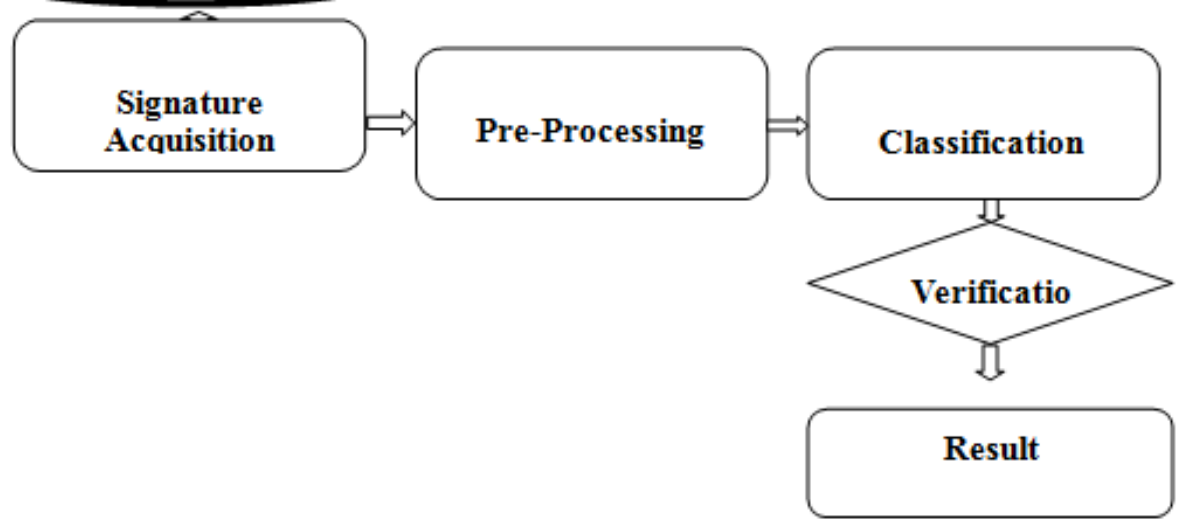

Figure 1. Flow Chart of Data

\subsection{Pseudo Code of the Proposed Technique}

i. Get signature image from the database.

ii. Pre-processing of images.

iii. Select important feature using the Principal Component Analysis. 
iv. Set a threshold to differentiate between the genuine and forged signature.

v. Classify signatures based on the threshold value.

\subsection{Graphical Representation of Proposed Technique}

The graphical representation of the model is provided in Figure 2.

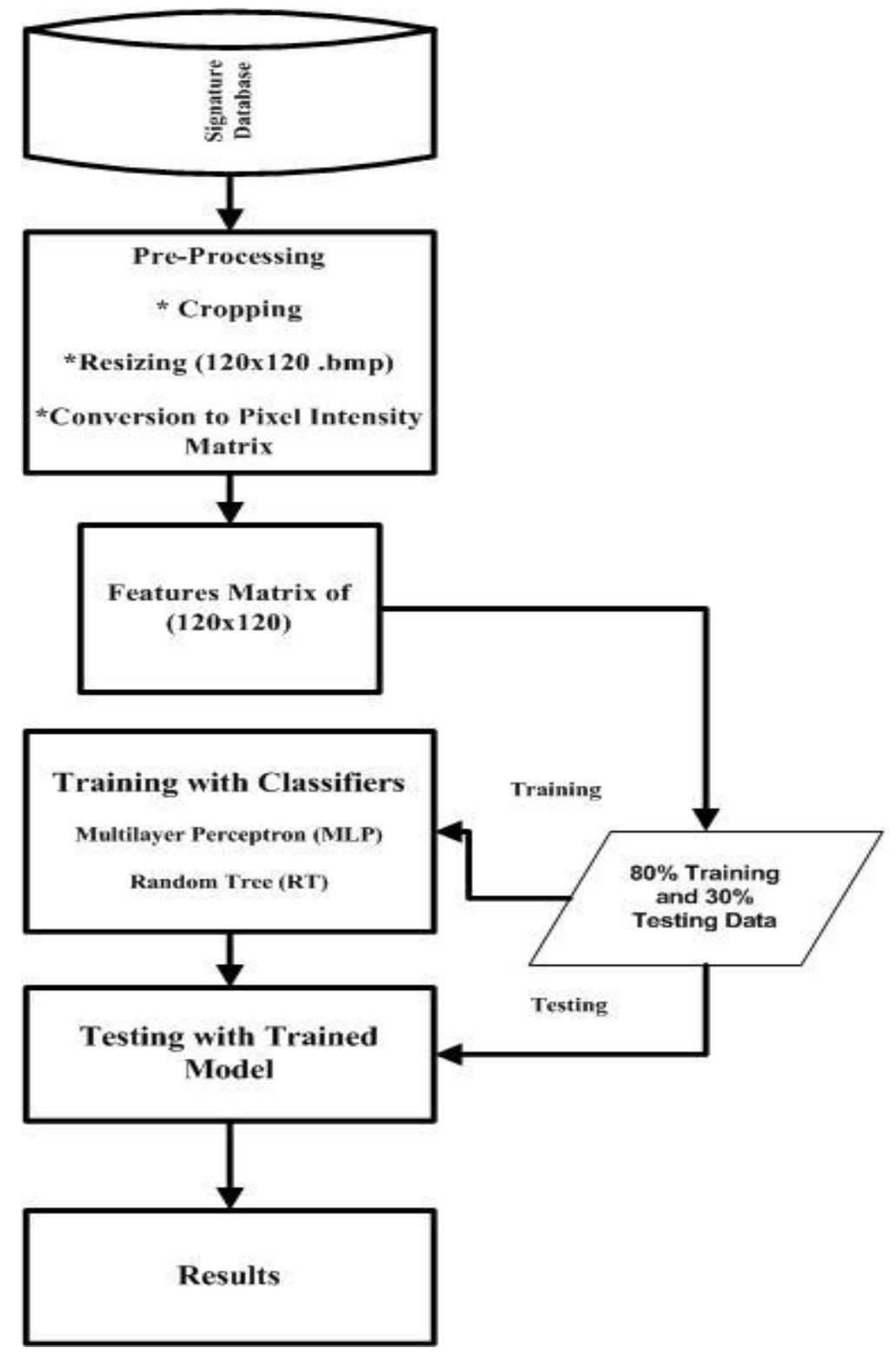

Figure 2. Graphical Representation of Proposed Model 


\section{Implementation of the Model}

The detailed implementation of the proposed model is provided here, the steps are explained as per steps of the above Figure 1.

\subsection{Signature Database}

The database used for experimentation was offline signature sub-corpus of MINISTERIO DE CIENCIA Y TECHNOLOGIA (MCYT) database containing signatures of 75 individuals from different Spanish sites. The signature sub-corpus contains 15 genuine and 15 forgeries against each signer and total of 2250 signatures [13$14]$.

\subsection{Signature Acquisition}

The offline signatures can be acquired by different sources like some authors have generated their own databases by getting signatures from individuals and generated skilled forgeries of the same as well. In this paper, we have acquired the signatures from the already available MCYT database which is freely available for non-commercial research. The signatures are selected randomly for the verification and identification.

\subsection{Pre-Processing}

The preprocessing step has been carried so that the signature images can be converted into the .arff file which is recognizable to Weka. The cropping of the signature has been carried out with Matlab and for conversion into the .arff file, the notepad has been used.

\subsection{Classification}

In previous research [2] we have used three classifiers, the Naïve Bayes Tree, Decision Tree J48, and k-Nearest Neighbor and achieved comparatively better results. In this paper, we have used the same technique of the [2] and applied Random Forest and Multilayer Perceptron with the signatures to get better results. The detailed description of the classifiers is provided in Sub-sections 3.4.1, and 3.4.2.

\subsubsection{Random Forest}

Random Forest classifier is the most powerful ensemble classifier of the decision tree applied in pattern recognition and classification. To overcome the high variance in decision tree classifier decision forest methodology was proposed by [15]. It can be considered as a classifier containing different classification methods or one method having several parameters. According to [15], the ensemble classifiers h1(x), h2(x),.. $\mathrm{hk}(\mathrm{x})$, and training set drawn from random vector $\mathrm{X}, \mathrm{Y}$, the margin function can be calculated by (1).

$$
\operatorname{margin}(X, Y)=\text { avk } I(h k(X)=Y)-\max J \neq Y \text { avk } I(h k(X)=J)
$$

According to [15], the larger the margin indicates more confidence in the classification. The generalization error can be calculated by (2).

$$
P E^{*}=P X_{s} Y(m g(X, Y)<0)
$$

Suppose we have a learning set $\mathrm{L}=((\mathrm{m} 1, \mathrm{n} 1), . .,(\mathrm{mi}, \mathrm{ni}))$ with $\mathrm{I}$ vectors with $\mathrm{m} \in \mathrm{X}$ and $n \in Y$ where $X$ represents the observations and $Y$ represent the class labels. For the prediction of a class, the classifier performs the mapping of $\mathrm{X} \rightarrow \mathrm{Y}$. Each individual tree in the forest categorizes an instance. 


\subsubsection{Multilayer Perceptron}

Multilayer Perceptron is a network of computation nodes known as Neurons or Perceptron. In the proposed method, MLP with an input layer, hidden layer, and output layer has been used, to classify the genuine and forgery offline signatures [16]. The MLP is a linear classifier which uses supervised learning technique, to convert the set of inputs to the set of output. The classification is based on linear predictor function, which combines a set of weights with the input vector. The Perceptron has different layers, for solving computational problems. The layers are connected with each other through the directed graph. For the training of the MLP, we have used back-propagation technique [17]. The training time was selected as 500s and threshold of 20. The Perceptron calculates a single output from multiple inputs by making a linear combination according to input weights and some nonlinear activation function which can be expressed by (3).

$$
\mathrm{Y}=\mathrm{F}(\mathrm{x})={ }_{\varphi}\left(\sum_{j=1}^{n} w_{j} x_{j}+b\right)={ }_{\varphi}\left(w^{T} \mathrm{X}+\mathrm{b}\right)
$$

Where, $y=F(x)$ is the Output, ' $w$ ' is the Weight Vector, ' $x$ ' is Input vector, $b=$ Biasness $_{\varphi}=$ Activation function.

\section{Experimentation}

Experimentation was carried out with Matlab R2015a and Weka 3.6. The preprocessing of signature images was carried out with Matlab and after pre-processing the signature images were classified with Weka 3.6.

\subsection{Results}

The results are calculated with 10 fold cross validation and $80 \%$ split, the detailed results are presented in the Table. 1.

Table 1. Classification Results

\begin{tabular}{|c|c|c|c|c|}
\hline \hline $\begin{array}{c}\text { No of } \\
\text { Users }\end{array}$ & \multicolumn{5}{|c|}{ Random Forest } & Multilayer Perceptron (MLP) \\
\hline \multicolumn{5}{|c|}{ 10 Fold Cross Validation (without forgery) } \\
\hline \hline & Accuracy & Error Rate & Accuracy & Error Rate \\
\hline 5 & $85.93 \%$ & $14.07 \%$ & $78.57 \%$ & $21.43 \%$ \\
\hline 10 & $87.20 \%$ & $12.80 \%$ & $67.83 \%$ & $32.17 \%$ \\
\hline Average & $\mathbf{8 6 . 5 6 \%}$ & $\mathbf{1 3 . 4 4 \%}$ & $\mathbf{7 3 . 2 0 \%}$ & $\mathbf{2 6 . 8 0 \%}$ \\
\hline \multicolumn{5}{|c|}{$\mathbf{1 0}$ Fold Cross Validation (with forgery) } \\
\hline \hline 5 & $76.40 \%$ & $23.60 \%$ & $66.73 \%$ & $33.27 \%$ \\
\hline 10 & $77.35 \%$ & $22.65 \%$ & $57.98 \%$ & $42.02 \%$ \\
\hline Average & $\mathbf{7 6 . 8 7 \%}$ & $\mathbf{2 3 . 1 3 \%}$ & $\mathbf{6 2 . 3 5 \%}$ & $\mathbf{3 7 . 6 5 \%}$ \\
\hline \hline \multicolumn{5}{|c|}{$\mathbf{8 0 \%}$ Training and 20\% Testing (without forgery) } \\
\hline 5 & $84.67 \%$ & $15.33 \%$ & $78.33 \%$ & $21.67 \%$ \\
\hline 10 & $86.67 \%$ & $13.33 \%$ & $69.42 \%$ & $30.58 \%$ \\
\hline Average & $\mathbf{8 5 . 6 7 \%}$ & $\mathbf{1 4 . 3 3 \%}$ & $\mathbf{7 3 . 8 7 \%}$ & $\mathbf{2 6 . 1 3 \%}$ \\
\hline \multicolumn{5}{|c|}{$\mathbf{8 0 \%}$ Training and 20\% Testing (with forgery) } \\
\hline 5 & $74.79 \%$ & $25.21 \%$ & $65.73 \%$ & $34.27 \%$ \\
\hline 10 & $76.61 \%$ & $23.39 \%$ & $58.13 \%$ & $41.87 \%$ \\
\hline Average & $\mathbf{7 5 . 7 0 \%}$ & $\mathbf{2 4 . 3 0 \%}$ & $\mathbf{6 1 . 9 3 \%}$ & $\mathbf{3 8 . 0 7 \%}$ \\
\hline \hline
\end{tabular}




\subsection{Results Comparison}

The results achieved with the Random Forest and Multilayer Perceptron (MLP) are compared with the results achieved in [18], by using Kernel Perceptron and SMO algorithm. The authors have achieved an accuracy of $73.36 \%$ with Kernel Perceptron and $71.19 \%$ accuracy with SMO algorithm. The results achieved with random forest are much better than the results achieved by [18], but there is a difference in the number of signature images. The authors of [18] have used a large number of signatures as compared to the number of signatures we have considered. The results achieved with the proposed method are compared with [19] in which the authors have achieved 57.00\% accuracy with Energy Density Method, accuracy $70.00 \%$ with Directional Features and $71.00 \%$ Directional Features with the Energy Density method by using 10 signature samples. The results achieved with Random Forest using 10 signatures are better as compared to the results of [19]. The authors of [20] have achieved an accuracy of $76.22 \%$ and $77.87 \%$, so the results we have achieved using Random Forest are comparatively better than the [20].

The results achieved with the Random Forest and Multilayer Perceptron (MLP) are compared with the results we have achieved with the Naïve Bayes Tree, Decision Tree J48, and k-Nearest Neighbor in [2]. Random Forest has proved an accuracy of $76.40 \%$ and error rate of $23.60 \%$ with 5 signatures with forgery, and the accuracy improved to $77.35 \%$ with the reduced error rate of $22.65 \%$. The accuracy rate of Random Forest is better than the accuracy rate of the Naïve Bayes Tree and Decision Tree J48. The detailed comparison of the results is provided in the Table. 2.

Table 2. Results Comparison with Already Proposed Models

\begin{tabular}{|c|c|c|c|c|c|}
\hline $\begin{array}{c}\text { Author/ } \\
\text { Reference }\end{array}$ & $\begin{array}{l}\text { No of } \\
\text { Users }\end{array}$ & $\begin{array}{l}\text { Dataset } \\
\text { Used }\end{array}$ & Technique used & $\begin{array}{l}\text { Error } \\
\text { Rate }\end{array}$ & Accuracy \\
\hline \multicolumn{6}{|c|}{ Offline Signature Verification Models } \\
\hline \multirow{2}{*}[16]{} & - & \multirow[t]{2}{*}{ - } & Kernel Perceptron & $26.64 \%$ & $73.36 \%$ \\
\hline & - & & SMO algorithm & $28.81 \%$ & $71.19 \%$ \\
\hline \multirow{3}{*}[17]{} & - & \multirow{3}{*}{ - } & Energy Density Method & $43.00 \%$ & $57.00 \%$ \\
\hline & - & & Directional Features & $30.00 \%$ & $70.00 \%$ \\
\hline & - & & $\begin{array}{l}\text { Directional Feature with } \\
\text { Energy Density Method }\end{array}$ & $29.00 \%$ & $71.00 \%$ \\
\hline \multirow{2}{*}[18]{} & - & \multirow{2}{*}{ MCYT } & - & $23.78 \%$ & $76.22 \%$ \\
\hline & - & & - & $22.13 \%$ & $77.87 \%$ \\
\hline \multirow{6}{*}{ [2] } & 5 & \multirow{6}{*}{ MCYT-75 } & \multirow{2}{*}{$\begin{array}{l}\text { Pixel Intensity levels } \\
\text { Naïve Bayes Tree }\end{array}$} & $29.21 \%$ & $70.79 \%$ \\
\hline & 10 & & & $36.75 \%$ & $63.25 \%$ \\
\hline & 5 & & \multirow[t]{2}{*}{ Decision Tree J 48} & $41.79 \%$ & $58.21 \%$ \\
\hline & 10 & & & $46.95 \%$ & $53.05 \%$ \\
\hline & 5 & & \multirow[b]{2}{*}{ k-Nearest Neighbor } & $11.96 \%$ & $88.04 \%$ \\
\hline & 10 & & & $11.80 \%$ & $88.20 \%$ \\
\hline \multirow{4}{*}{$\begin{array}{l}\text { Proposed } \\
\text { Method }\end{array}$} & 5 & \multirow{4}{*}{ MCYT-75 } & \multirow[b]{2}{*}{ Random Forest } & $23.60 \%$ & $76.40 \%$ \\
\hline & 10 & & & $22.65 \%$ & $77.35 \%$ \\
\hline & 5 & & \multirow{2}{*}{$\begin{array}{c}\text { Multilayer Perceptron } \\
\text { (MLP) }\end{array}$} & $33.27 \%$ & $66.73 \%$ \\
\hline & 10 & & & $42.02 \%$ & $57.98 \%$ \\
\hline
\end{tabular}

\section{Discussion}

The Multilayer Perceptron MLP training time was high as compared to the Random Forest, but we managed to improve the results of MLP by making some changes in the preprocessing steps. The increase in the number of signatures will also increase the 
accuracy of the MLP as the MLP requires a large amount of data for training. The pruning has not been applied here. The MLP and Random Forest have faced a low memory heap problem with the large dataset to avoid this problem we have performed 5 fold cross validation with larger datasets (10 users with forgery).

The results of the Random Forest shown some improvements with 10 signatures, including forgery as compared to 5 signatures with forgery, it proves that if we increase the number of training samples the accuracy will also improve.

The results are not satisfactory as compared to the other techniques, but the improvement is possible so we will try to improve the results further in future work.

\section{Conclusion}

In the continuation of our previous research carried out in [1-2], we have used Multilayer Perceptron and Random Forest classifiers for the signature verification. The results achieved with Random forest are quite satisfactory, but the results achieved with Multilayer Perceptron are low as compared to other techniques. The changes in preprocessing steps and filtering may increase the accuracy of the system further. The results in comparison with other techniques are satisfactory. The random Forest has achieved an accuracy of $76.40 \%$ with 5 signatures and the accuracy increased to $77.35 \%$ with 10 signatures. The Multilayer Perceptron has proved an accuracy of $66.73 \%$ with 5 signatures and the accuracy reduced to $57.98 \%$ with 10 signatures. In future work, we will change the pre-processing steps and reduce the dimension of the data and try to improve the accuracy of the system.

\section{References}

[1] A.S.Shah, M.N.A.Khan and A.Shah, "An Appraisal of Off-Line Signature Verification Techniques," International Journal of Modern Education and Computer Sciences, vol.7, no.4, (2015), pp.67-75.

[2] A.S. Shah, M. N. A. Khan, F. Subhan, M. Fayaz, A. Shah, "An Offline Signature Verification Technique using Pixels Intensity Levels," International Journal of Signal Processing, Image Processing and Pattern Recognition, vol. 9, no.8, (2016), pp. 205-222.

[3] Jena.D, Majhi. B, Panigrahy.K, Jena.K.S (2008), Improved Offline Signature Verification Scheme Using Feature Extraction Method, Centre for IT Education, Bhudaneswar, 751010, Orissa, India, National Institute of Technology Rourkela, 769008, Orissa, India, Roland Institute of Technology, Berhampur, 761008, Orissa, India, 2008, $7^{\text {th }}$ IEEE in-conference, pp 475-480.

[4] https://en.wikipedia.org/wiki/Forensic_science

[5] M. Shah, A. S. Shah, and I. Ijaz, "Implementation of User Authentication as a Service for Cloud Network," International Journal of Grid and Distributed Computing (IJGDC), vol.9, no.10, (2016), 197 210.

[6] M. Shah and A. S. Shah, "Appraisal of the Most Prominent Attacks due to Vulnerabilities in Cloud Computing," International Journal of Grid and Distributed Computing (IJGDC), vol.9, no.7, (2016), pp.13-22.

[7] Bansal. A, Garg. D, Gupta. A (2008), A pattern Matching Classifier for offline Signature verification, Computer Engineering, NSIT, Delhi, India, IEEE, pp 1160-1163.

[8] H. K. Khan, A.S. Shah and M. A. Khan, "Critical Evaluation of Frontal Image-Based Gender Classification Techniques", International Journal of Signal Processing, Image Processing and Pattern Recognition, vol. 9, no.10, (2016), pp. 313-324.

[9] Liu.L, Duan.H (2009). The Research of Handwritten Signatures, ITME2009 - Proceedings 2009 IEEE International Symposium on IT in Medicine and Education. pp 1066-1069.

[10] Y.Serdouk, N.Hassiba, and C. Youcef, "New Gradient Features for Off-Line Handwritten Signature Verification", 2015 International Symposium on Innovations in Intelligent Systems and Applications (INISTA), (2015).

[11] Bertolini, L.S.Oliveira, E.Justino, and R.Sabourin, "Reducing Forgeries in Writer-Independent Off-Line Signature Verification through Ensemble of Classifiers", Pattern Recognition, vol. 43, (2010), pp. 387396.

[12] Miguel-Hurtado. O , Mengibar-Pozo. L , Pacut. R, (2008). A new algorithm for signature verification system based on DTW and GMM, University Carlos III of Madrid, IEEE, pp: 206-213.

[13] J.Fierrez-Aguilar, N.Alonso-Hermira, G. Moreno-Marquez and J. Ortega-Garcia, "An off-Line Signature Verification System Based on Fusion of Local and Global Information", In Biometric Authentication, (2004), pp. 295-306. 
[14] J. Ortega-Garcia, J.Fierrez-Aguilar, D.Simon, J.Gonzalez, M.Faundez-Zanuy, V.Espinosa, A.Satue., "MCYT Baseline Corpus: a Bimodal Biometric Database", IEE Proceddings-Vision, Image and Signal Processing, vol. 150, no. 6, (2003), pp. 395-401.

[15] L. Breiman "Random forests", Machine Learning, vol. 45, no. 1, (2001), pp. 5-32.

[16] S. Odeh, and M. Khalil, "Apply Multi-Layer Perceptron Neural Network for Off-Line Signature Verification and Recognition", International Journal of Computer Science Issues, vol.8, no. 6, (2011), pp. 261-266

[17] B.H. Shekar, and R.K. Bharathi, "Off-Line Signature Verification Based on Principal Component Analysis and Multi-Layer Perceptrons", volume 235 of the series Advances in Intelligent Systems and Computing, (2014), pp. 101-109.

[18] C. Kruthi and D.C. Shet, "Offline Signature Verification Using Support Vector Machine", 2014 Fifth International Conference on Signal and Image Processing (ICSIP), (2014), pp. 3-8.

[19] M. Tomar and P. Singh, "A Directional Feature with Energy Based Offline Signature Verification Network", International Journal on Soft Computing, vol.2, no.1, (2011), pp. 48-57.

[20] F.Alonso-Fernandez, M.C. Fairhurst, J.Fierrez and J.Ortega-Garcia, "Automatic Measures for Predicting Performance in Off-Line Signature", IEEE International Conference on, Image Processing, vol. 1, (2007), pp. 369-372.

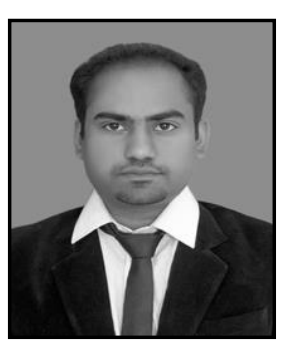

Authors

Abdul Salam Shah, is currently doing specialization in Management Information System (MIS) from Virtual University of Pakistan. He has completed MS degree in Computer Science from SZABIST, Islamabad, Pakistan in 2016. He did his BS degree in Computer Science from Isra University Hyderabad, Sindh Pakistan in 2012. In addition to his degree, he has completed short courses and diploma certificates in Databases, Machine Learning, Artificial Intelligence, Cybercrime, Cybersecurity, Networking, and Software Engineering. He has published articles in various journals of high repute. He is a young professional and he started his career in the Ministry of Planning, Development and Reforms, Islamabad Pakistan. His research area includes Machine Learning, Artificial Intelligence, Digital Image Processing and Data Mining. Mr. Shah has contributed in a book titled "Research Methodologies; an Islamic perspectives," International Islamic University Malaysia, November, 2015.

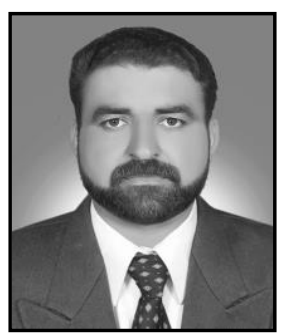

Masood Shah, enthusiastic and high-achieving IT professional, has completed MS degree in Computer Science from SZABIST, Islamabad, Pakistan in 2016. He did his Bachelor of Information Technology from Agricultural University, Peshawar Pakistan in 2012. He has completed short courses and diploma certificates in CCNA (Cisco Certified Network Associate), MCSE (Windows Server 2008), Cybercrime, Cyber Security, Networking. He is a young professional having exceptional technical and analytical skills, with over 3 years' experience of Computer System/ Network Administration, Information System Support \& Security, Network and Server support. He has worked with Techno-ed Pvt Ltd Islamabad, Money Link Exchange Peshawar, and Waseela-e-Taleem - Benazir Income Support Programme. He is currently working as Lecturer with Frontier Comprehensive School \& College (FCS), Shergarh, Pakistan. His research area includes Cloud Computing, Cyber Security, and Cryptography. 


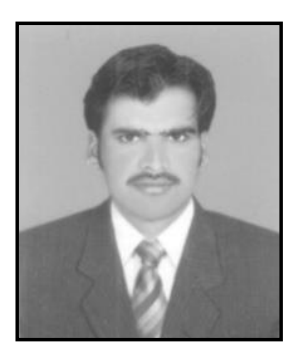

Muhammad Fayaz, is currently perusing Ph.D. in Computer Science. He received MS in Computer Science from SZABIST, Islamabad, Pakistan in 2014. He did MSC from the University of Malakand, KPK, Pakistan in 2011. His areas of interest are NP problems, Approximation Algorithms, Image Processing, and Pattern Recognition.

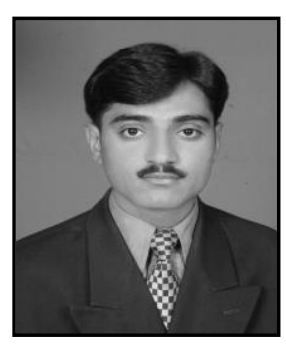

Fazli Wahid, received BS in Computer Science from University of Malakand, Pakistan in 2006, and MS in Computer Science from SZABIST, Islamabad, Pakistan in 2015. His area of interest are energy consumption prediction, optimization, and management using multilayer perceptron, Artificial Bee Colony, Ant Colony and other Machine Learning Techniques.

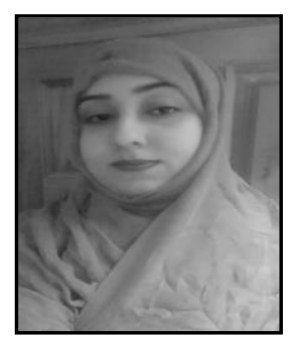

Hira Khalid Khan, has completed MS degree in Computer Science from SZABIST, Islamabad, Pakistan in 2016. She has received BS degree in Computer Science from University of Science and Technology, Bannu, Khyber Pakhtunkhwa, Pakistan in 2012. Besides her academic qualification, she has attended training of Microsoft Experts Systems, Under Directorate of Information Technology, Government of Khyber Pakhtunkhwa, Peshawar, Pakistan in 2013. Her research area includes, Machine Learning, Artificial Intelligence, Pattern Recognition, and Digital Image Processing.

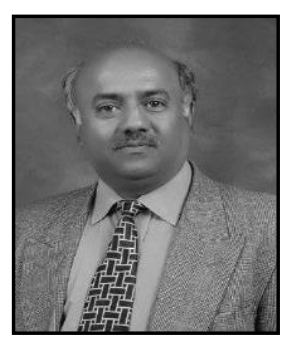

Asadullah Shah, is working as Professor and Head of department of Information Systems (HOD) at the Kulliyyah of ICT, International Islamic University Malaysia (IIUM) before joining IIUM, he worked as Head of Telecommunication Engineering \& Management department, IoBM Karachi Sindh, Dean Faculty of Computer and Management Sciences, Isra University Hyderabad Sindh and Head of Telecommunication Engineering and IT, Sukkur IBA, Sindh-Pakistan. He did his Ph.D. from the university of Surrey UK, in 1998, with the specialization in Multimedia Communication. He started his academic carrier from University of Sindh Jamshoro, Pakistan in 1986 as a lecturer. He has published 200 research articles in highly reputable international and national journal in the field of computers, communication and IT. Also, he has published 12 books in his 30 years of the academic carrier. Currently he is supervising great number of postgraduate students, working in multiple disciplines, specially, animation, social media and image processing in the Department of Information Systems, Kulliyyah of Information and Communication Technology, International Islamic University Malaysia.

\footnotetext{
*Corresponding Author
} 